\title{
Insegnare la Shoah. È un monito per il futuro?
}

\author{
di Carla Marcellini
}

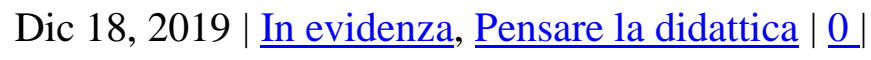

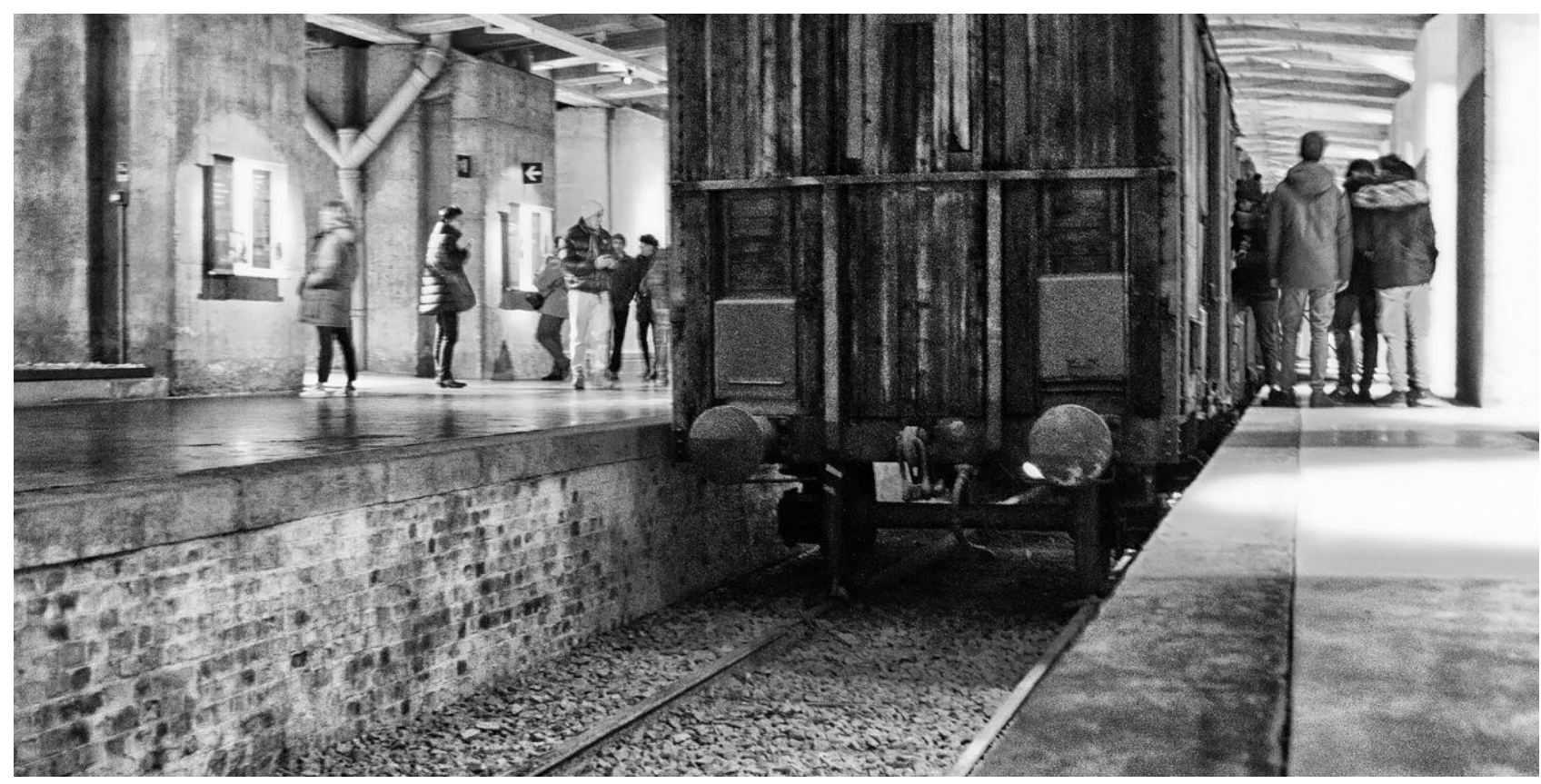

Vagoni al Binario 21 (Memoriale della Shoah).

Foto di Settimioma - Own work, CC BY-SA 4.0, $\underline{\text { Link }}$

\begin{abstract}
L'articolo affronta il tema dell'insegnamento della Shoah nella scuola italiana. L'analisi delle pratiche didattiche più diffuse, come l'incontro con il testimone e la visita ai luoghi, permette di avviare una riflessione intorno al rapporto tra memoria e storia, agli usi potenzialmente strumentali della storia della Shoah e alla necessità di ricondurre al centro dell'insegnamento il docente e lo storico, figure essenziali alla comprensione del passato e alla costruzione del sapere disciplinare.
\end{abstract}

\section{Introduzione}

Il contributo[1] intende proporre alcune riflessioni su come, dall'istituzione del Giorno della Memoria (legge 211 del 20 luglio 2000), nella scuola italiana sia stato affrontato il tema della Shoah. Lo sguardo è rivolto alla scuola, i cui attori sono in primo luogo gli insegnanti, ma anche alle associazioni e istituzioni che in collaborazione con le scuole organizzano approfondimenti dedicati.

Che tipo di insegnamento ha prevalso fino a ora, come si è lavorato, che cosa si è fatto, con quali obiettivi, in che modo? Si tratta necessariamente di riflessioni che guardano alla generalità degli interventi educativi, agli approcci di tendenza più che al lavoro dei singoli insegnanti in classe.

Per fare questo si richiamano alcuni concetti che sono alla base dell'insegnamento della storia e parte integrante delle competenze professionali di un docente: "storia", "memoria individuale", "memoria pubblica", "commemorazione". Occorre aggiungere che la riflessione nasce anche da un certo sconforto dovuto ai numerosi e ripetuti episodi di razzismo e di antisemitismo in crescita, 
anche in paesi come l'Italia, in cui le politiche memoriali hanno avuto ampia diffusione nel sistema educativo.

La legge ha senz'altro reso visibile la tragedia della Shoah, con l'obiettivo ben esplicitato, di «conservare nel futuro dell'Italia la memoria di un tragico ed oscuro periodo della storia nel nostro Paese e in Europa e affinché simili eventi non possano mai più accadere». L'inizio del XXI secolo aveva portato la consapevolezza di come gli italiani si fossero arroccati su un racconto del fascismo e della guerra in cui evidenti e gravi "vuoti di memoria"[2] rischiavano di falsare la verità storica. La convinzione che la Shoah fosse responsabilità esclusiva della Germania di Hitler e quasi un incidente di percorso della storia europea, era prevalente, talvolta anche nei manuali scolastici, sulla certezza storica delle responsabilità del regime fascista.

\section{Percezioni}

Qualche anno fa mi hanno invitato a parlare in un liceo classico in occasione del Giorno della Memoria. Successivamente al mio intervento vi sarebbe stato un gruppo musicale chiamato a eseguire brani di musica Klezmer. Entrando in Aula magna mi sono trovata in mezzo al flusso degli studenti che arrivavano per sistemarsi nei posti a sedere. Nella confusione generale ho ascoltato un dialogo. Il primo studente chiedeva all'amico che cosa si andasse a fare e l'altro candidamente rispondeva: è la festa degli ebrei. Questo episodio che risale al 2008, per la prima volta mi ha costretto a riflettere su come nella scuola si stesse ottemperando alla prescrizione della legge 211. Gli episodi di intolleranza e di antisemitismo che popolano le cronache nazionali e che nel 2008 erano numericamente inferiori rispetto a oggi, sono un altro elemento di cui occorre tenere conto per provare a capire se esista una qualche relazione tra quanto viviamo e quanto abbiamo fatto per scongiurare l'intolleranza, il razzismo, la violenza.

Lo studente che risponde "è la festa degli ebrei" non ha capito il significato del Giorno della memoria, che non è un atto di omaggio agli ebrei uccisi, poiché non è per loro che si celebra il 27 gennaio e neppure per gli ebrei di oggi, che non hanno bisogno di questa giornata per ricordare quanto accadde. È per tutti gli altri, perché lo sterminio e la deportazione sono stati una tragedia europea in cui gli ebrei furono le vittime e non i protagonisti.

Negli ultimi anni colgo, nel sentire comune, una duplice tendenza in occasione di questa data. Da un lato si manifesta in alcuni un certo senso di insofferenza, come se questo celebrare ogni anno il ricordo di quei drammatici eventi ne abbia affievolito il senso, appiattendo il passato sulla ritualità. Dall'altro c'è una sorta di "dovere di memoria", che impone a scuole, enti e istituzioni di fare comunque qualcosa per non perdere questo appuntamento. La cosa è ancora più evidente nei social network in cui il presenzialismo impone di postare immagini, articoli, riflessioni, pensieri, pur di non far passare questa data senza aver condiviso un gesto di visibilità.

Sono due atteggiamenti apparentemente contraddittori che forse trovano senso nel tipo di approccio che si è avuto rispetto a questa data. Mi sembra, infatti, che molto si sia lavorato per la memoria della Shoah, ma non abbastanza per comprenderne la storia, le dinamiche, il contesto in cui è nata, le responsabilità dei singoli, degli stati, della cultura, della società e della politica.

\section{La memoria e la storia}


La memoria e la storia richiamano contesti diversi anche se strettamente legati l'uno all'altro. La memoria è un atto soggettivo o collettivo di ricostruzione del passato: «selettivo e deliberato, e non un deposito indifferente di ricordi accumulati nel tempo» dice David Bidussa[3]. La memoria è un esercizio per tenere a bada il disordine, è un'elaborazione ordinata del reale; essa non è la registrazione di tutto il nostro passato, ma solo di quello che è ritenuto coerente con il nostro presente. In quanto atto soggettivo è sempre la memoria di un singolo o di un gruppo; il suo radicamento nella dimensione esistenziale la rende "vera" e indiscutibile agli occhi del soggetto che ricorda.

La storia, invece, non ha come obiettivo ricordare, ma capire e interpretare. È un processo di ricostruzione attuato con rigore basato sull'uso delle fonti (tra cui possono anche entrare le testimonianze). Nella memoria esiste una volontà di coerenza e identità raggiunta attraverso emotività ed empatia, mentre la storia va oltre la vicenda esistenziale ed emotiva del/dei soggetto/i, per cercare di spiegare, capire, ricostruire il contesto e le dinamiche del passato. Chi c'era, chi ha vissuto certe esperienze dolorose e drammatiche, ha dovuto necessariamente cercare di capirle per dare un senso alla propria vicenda; tuttavia da questa tipologia di analisi retrospettiva si apre uno sguardo parziale e soggettivo, aggettivi che non appartengono alla ricostruzione storica che ha un progetto molto più ambizioso, ampio, complesso, tendenzialmente oggettivo e inclusivo.

Per la logica memoriale l'accesso alla testimonianza è sufficiente a spiegare e comprendere cosa sia accaduto e non vi è dubbio che l'approccio emotivo che si genera dall'ascolto di un testimone è un punto di forza dell'insegnamento, sia in storia che in letteratura, perché riesce a catturare le corde più profonde. Ma il lavoro didattico non può fermarsi a questo: le memorie individuali appaiono il modo più semplice per coinvolgere gli studenti, eppure sono uno strumento difficile per l'insegnamento della storia[4], Esse andranno ricondotte all'interno di una narrazione più vasta e di una storia molto più complessa per allontanare la possibilità che lo sguardo soggettivo del testimone diventi storia.

\section{La memoria pubblica e la commemorazione}

La memoria pubblica è un patto non esplicitato, che sancisce cosa una comunità trattenga del proprio passato a fondamento della convivenza per il futuro. È un'operazione di selezione da cui scaturiscono spesso ritualità che finiscono per essere commemorazione di eventi del passato, pratica assai diffusa per il 27 gennaio. Proprio la ritualità vale come verifica tangibile dell'identità di un popolo o di una comunità. Un po' com'è accaduto per il centocinquantesimo dell'unità d'Italia: «il momento in cui i cittadini constatano di avere pensieri e valori comuni»[5]. La commemorazione si muove sul piano dei valori morali e della religione civile ed è uno degli strumenti attraverso i quali si costruisce la memoria pubblica.

Le istituzioni - e la scuola in primis - come tutti coloro che si occupano di organizzare iniziative in occasione del Giorno della memoria, dovrebbero operare con consapevolezza e saper distinguere tra fare storia, ricordare, commemorare. È infatti importante riflettere su che tipo di intervento educativo si voglia fare e con quali obiettivi.

I testimoni della Shoah: le persone 
Come si è lavorato in quasi venti anni nelle scuole e con le scuole? Si è iniziata a diffondere la conoscenza sul genocidio attraverso i testimoni, i quali hanno svolto un ruolo importantissimo di sensibilizzazione verso un tema di cui non si parlava. Tuttavia, spesso, il lavoro didattico iniziava e si concludeva con il racconto personale del testimone o perlomeno la continuazione del lavoro didattico, appena iniziato, era lasciata alla volontà e alle competenze del singolo insegnante, nella convinzione che fare appello all'emotività potesse di per sé mettere in atto processi di conoscenza e di comprensione. La scuola, e in particolare gli insegnanti di storia, si sono spesso trovati impreparati a lavorare sulla memoria: era la prima volta che in classe entravano i protagonisti della Shoah. Certamente l'esperienza dell'ascolto e di sentirsi parte della storia, ha determinato coinvolgimento e lasciato in chi ascoltava sentimenti intensi. Tuttavia non esiste nessun automatismo che parta dall'emozione per giungere alla comprensione. Occorre la mediazione del lavoro didattico e probabilmente non sempre si è intrapresa la strada che dalla memoria delle fonti orali porta alla comprensione del passato e alla costruzione del sapere storico.

\section{I testimoni della Shoah: i luoghi}

David Bidussa nel 2009 scriveva:

Una volta che le voci testimoniali di un evento scompariranno che cos'avremo in mano? Come elaboreremo quel vuoto? E allo stesso tempo come rifletteremo? La questione riguarda la capacità che quelle voci hanno di parlare e di suscitare domande; non solo di riprodurre se stesse. In quel terreno vuoto si porrà la dimensione della postmemoria, di una riflessione che vivrà unicamente e strutturalmente della capacità di elaborare documenti.

Lì si porrà il problema del rapporto tra testimonianza e storia. Quando i testimoni oculari saranno scomparsi, quando quelle voci non avranno più voce, ci ritroveremo con un archivio definito di storie, che racconteranno scenari e situazioni. Si tratterà allora di far lavorare quelle storie narrate come «documenti»[6].

Allo scomparire físiologico dei testimoni, si è aperta davvero l'era della postmemoria? Davvero quelle narrazioni sono diventate «documenti»?

A guardare bene nel mondo della scuola questo passaggio non è ancora avvenuto. Sembra che invece di rivolgere lo sguardo laddove si possono avere strumenti per capire - e non solo per conoscere - non si sia abbandonato quell'approccio emotivo e sofferto che l'incontro con il testimone rendeva inevitabile, e al contempo ne costituiva il limite.

Si sta diffondendo la pratica di andare nei luoghi della memoria della Shoah con viaggi organizzati da enti pubblici e privati, infatti negli ultimi dieci anni migliaia di studenti e docenti hanno visitato quei luoghi. Senza voler demonizzare e neppure irridere tale pratica apostrofata da alcuni, con tono critico, "turismo della memoria", tuttavia il viaggio sembra essere diventato il modo per sostituire i testimoni o, perlomeno, sembra ispirarsi alle stesse modalità di approccio educativo.

Il viaggio viene spesso considerato una sorta di modello di insegnamento che "chiavi in mano" offre la possibilità alle scuole di adempiere al dovere della memoria, svolgere la parte del programma di storia a cui si riferisce, affidandosi ad esperti competenti e preparati. La preparazione delle classi avviene con una serie di lezioni e attività propedeutiche. 
La tendenza più diffusa è l'idea che il viaggio ad Auschwitz o nei luoghi della Shoah possa sostituire o semplificare il lavoro in classe di sistematica costruzione del sapere storico, condotta attraverso fonti, brani di storiografia e manuale scolastico. Uno degli elementi più critici di questa modalità sta nel fatto che per comprendere si parta dal punto finale, con il rischio di comunicare, implicitamente, che ciò che è accaduto prima di Auschwitz sia stato meno grave. La mancata comprensione del processo politico e culturale che sta alle spalle della tragedia rende la Shoah un evento esemplare della storia del Novecento, in nome della sua unicità. Scrive Valentina Pisanty[7]:

La sacralizzazione è un dispositivo ideologico, non importa quanto consapevole e intenzionale, mirato a sottrarre un evento - nella fattispecie la Shoah - al suo contesto storico specifico [...], semplificandone la rappresentazione e preservandola da incursioni indesiderabili attraverso un sistema di divieti (la cui inosservanza espone l'aggressore all'ostracismo), evidentemente allo scopo di rivendicare un monopolio sulla scelta delle interpretazioni e degli usi a cui la memoria di tale evento può dare adito.

Sui concetti di sacralizzazione e banalizzazione della memoria della Shoah, le pagine di Valentina Pisanty sono illuminanti. Auschwitz sembra essere diventata la rappresentazione del male assoluto, assumendo quasi un valore simbolico di paradigma rovesciato dei diritti e dei valori umani. Mostrare il luogo del male sembra, così, essere l'antidoto alla tragedia, tuttavia si tratta di un'ingenuità dal punto di vista didattico. Se bastasse vedere per capire, allora probabilmente le tante drammatiche immagini che quotidianamente giungono agli occhi degli studenti dovrebbero essere sufficienti a sensibilizzare contro le tragedie dell'immigrazione, le guerre in Medio Oriente, gli scenari di sopraffazione e sfruttamento di popoli e individui. Eppure non è così. Le immagini scorrono davanti agli occhi e sembrano assuefare alle tragedie altrui.

Il rischio che si corre anteponendo le immagini dei luoghi alla formazione storica è il medesimo. La convinzione di fondo che sta dietro questo approccio conoscitivo è che vedere il luogo della messa a morte conti più di qualsiasi lezione di storia, come se guardare equivalesse a conoscere e comprendere. Eppure, solo quest'ultimo è l'obiettivo della storia e del suo insegnamento. Si può imparare e comprendere senza vedere. Le immagini e la visita dei luoghi dovrebbero collocarsi successivamente rispetto allo studio delle dinamiche storiche, politiche e culturali, del contesto in cui quei fatti sono avvenuti[8].

Immedesimarsi nelle storie altrui attraverso il racconto o la visita ai luoghi non è che una parte di un lavoro mirato a spiegare gli snodi, i passaggi, i processi storici e politici che sono alla base della individuazione delle responsabilità storiche dei singoli attori. La memoria del genocidio non è unica e omogenea, non è memoria esemplare dell'orrore e della tragedia, non può essere retorica del male assoluto. Solo lo studio della storia può restituire le singole responsabilità anche a partire da un processo di immedesimazione soggettiva rispetto alle storie non solo delle vittime, ma anche dei carnefici. In questa prospettiva, il libro intervista di Gitta Sereny[9] al boia di di Treblinka, Franz Stangl è uno strumento prezioso dal punto di vista didattico perché progressivamente vi viene ricostruito il contesto personale e sociale che rese possibile dirigere un campo di sterminio. «D'altra parte - come sostiene Georges Bensoussan - è solo attraverso la ragione che bisogna tentare di chiarire questo inferno, descriverne le forme e i processi, e dimostrare come una civiltà abbia potuto inscrivere il crimine nel suo funzionamento quotidiano»[10].

\section{È davvero un monito per il futuro?}


La volontà di trasmissione collettiva della Shoah ha spesso piegato il contenuto e la qualità del suo insegnamento alla necessità di divulgazione di massa; tanto che spesso sembra che si sia di fronte a un fenomeno culturale $\mathrm{o}$ a un rito sociale, più che alla ricostruzione di uno dei momenti più drammatici della storia del secolo scorso.

L'amplificazione mediatica, la mobilitazione nazionale, il monito a dover ricordare finisce per produrre forme di conoscenza semplificata e sintetica, immettendo in circolo sempre le stesse immagini e gli stessi schemi di pensiero, in cui ogni passaggio rinvia al successivo, come un automatismo che tende a riprodurre una sorta di dispositivo educativo: l'immagine dell'orrore e della sofferenza, il peso morale della testimonianza, il dovere di memoria, il monito per il futuro, la predica morale sulla tolleranza e sui diritti umani da rispettare[11].

La storia della Shoah diventa spesso lo strumento attraverso il quale si affronta il tema della negazione dei diritti, con l'obiettivo di sensibilizzare i giovani ai valori della democrazia, della pace, della tolleranza e della libertà. È evidente che nella storia della Shoah tutti questi valori siano stati negati e calpestati. È altrettanto evidente che una delle finalità dell'insegnamento storico sia l'educazione alla cittadinanza. Tuttavia spesso il processo didattico che dovrebbe partire dalla conoscenza, passare per la comprensione e poi giungere all'acquisizione di competenze di cittadinanza di cui libertà, democrazia, responsabilità e partecipazione sono i fondamenti, venga in qualche modo abbreviato, a scapito dell'efficacia. Avviene quando la Shoah diventa strumento per parlare di diritti negati, di libertà violate, di democrazia calpestata nel mondo attuale. Avviene quando l'attualizzazione mette in moto un processo di museificazione della Shoah che diviene il paradigma del male assoluto, con il quale misurare i mali del presente, per fare in modo che tanto orrore non accada mai più.

Il processo di costruzione delle competenze di cittadinanza, passa in buona misura attraverso lo studio della storia: la complessità degli scenari storici che aprono domande, questioni non risolte, contraddizioni dell'agire umano, di cui il passato è costituito, sono passaggi essenziali. Costruire sapere storico è un lavoro paziente e difficile che va condotto passo dopo passo, cercando sempre di avere come obiettivo la comprensione e non la stigmatizzazione, la costruzione della complessità e non la semplificazione.

Il genocidio ebraico è una storia di persone: carnefici, vittime, spettatori. È una storia di uomini e donne che hanno scelto di dare morte o convivere con essa. È una storia che interroga da vicino la nostra quotidianità e la sfera della responsabilità. La dimensione empatica ed emotiva non è sufficiente a spiegarla. Essa può però essere lo strumento attraverso il quale ci si ponga interrogativi, cui lo studio dei processi storici può dare risposte.

Spesso è diffusa la convinzione che per insegnare il genocidio servano competenze particolari, sensibilità speciali, conoscenze specifiche. Questa è una delle ragioni per cui si delega ad altri il suo insegnamento. Come in ogni professione, nessuno ha maggiori competenze di chi ne è titolare. Quello dell'insegnante è un mestiere intellettuale. Non servano abilità particolari o diverse da quelle che servono per organizzare un buon insegnamento di storia: conoscenza dei fatti, rigore e precisione, uso delle fonti, conoscenza della storiografia, capacità di sollevare interrogativi attraverso narrazione e interpretazione. Dopo l'ultimo testimone ci sono molte figure: gli operatori, gli esperti, il sistema mediatico, il mondo della politica; ma le due figure essenziali senza le quali tutto il resto non produce comprensione, rimangono lo storico e l'insegnante. 
- G. Bensoussan, L'eredità di Auschwitz. Come ricordare?, Einaudi, Torino 2014.

- D. Bidussa, Dopo l'ultimo testimone, Einaudi, Torino 2009.

- Cataruzza, M. Flores, S. Levis Sullam, E. Traverso (a cura di), Storia della Shoah. La crisi dell'Europa, lo sterminio degli ebrei e la memoria del XX sec., vol. 4, Utet, Torino, 2005.

- M. Gigli, M. L. Marescalchi, Il laboratorio nei luoghi di memoria, in P. Bernardi, F. Monducci (a cura di), Insegnare storia. Guida alla didattica del laboratorio storico, Utet, Torino, 2012, seconda edizione.

- C. Marcellini, Testimoni a scuola. Una riflessione sull'uso delle fonti orali per la didattica della storia, in "Novecento.org", n. 3, 2014.

- V. Pisanty, Abusi di memoria. Negare, banalizzare, sacralizzare la Shoah, Bruno Mondadori, Milano, 2012.

- V. Pisanty, L'irritante questione delle camere a gas. Logica del negazionismo, Bompiani, Milano, 2014.

- S. Pivato, Vuoti di memoria. Usi e abusi della storia nella vita pubblica italiana, Laterza, Bari, 2007.

- G. Sereny, In quelle tenebre, Adelphi, Milano, 1994.

Note:

[1] L'articolo è la rielaborazione di un testo in via di pubblicazione nel numero 7 della collana Il tempo, la storia e la memoria, Eum, Macerata 2020.

[2] L'espressione è mutuata dal titolo del libro di S. Pivato, Vuoti di memoria. Usi e abusi della storia nella vita pubblica italiana, Laterza, Bari 2007.

[3] Sulla questione della memoria e della storia si rimanda al libro di D. Bidussa, Dopo l'ultimo testimone, Einaudi, Torino 2009, in particolare il capitolo 6, Fare i conti con il passato, pp. 79-114.

[4] Sull'uso della memoria orale come fonte per la didattica della storia mi permetto di segnalare C. Marcellini, Testimoni a scuola. Una riflessione sull'uso delle fonti orali per la didattica della storia, in "Novecento.org", n. 3, 2014, <http://www.novecento.org/pensare-la-didattica/testimoni-ascuola-una-riflessione-sulluso-delle-fonti-orali-per-la-didattica-della-storia-996/> (ultima consultazione 3 ottobre 2019).

[5] G. Bensoussan, L'eredità di Auschwitz. Come ricordare?, Einaudi, Torino 2014, p. 143.

[6] Bidussa, 2009 p. 5.

[7] V. Pisanty, Abusi di memoria. Negare, banalizzare, sacralizzare la Shoah, Bruno Mondadori, Milano 2012, pp. 89-90.

[8] Circa la didattica dei luoghi di memoria mi limito a segnalare M. Gigli, M. L. Marescalchi, Il laboratorio nei luoghi di memoria, in P. Bernardi, F. Monducci (a cura di) Insegnare storia. Guida alla didattica del laboratorio storico, Utet, Torino 2012, seconda edizione, pp. 241-269.

[9] G. Sereny, In quelle tenebre, Adelphi, Milano1994. 
[10] Bensoussan, 2014 p. 83.

[11] Su questi temi si veda nello specifico Bensoussan, 2014, pp. 112-144. 